\title{
Contrast-Enhanced 3D-FLAIR Imaging of the Optic Nerve and Optic Nerve Head: Novel Neuroimaging Findings of Idiopathic Intracranial Hypertension
}

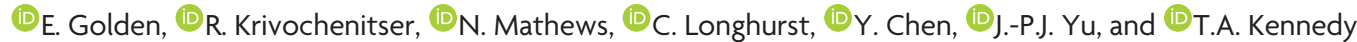

\begin{abstract}
BACKGROUND AND PURPOSE: The sensitivity of contrast-enhanced 3D-FLAIR has not been assessed in patients with idiopathic intracranial hypertension. The purpose of this study was to evaluate whether hyperintensity of the optic nerve/optic nerve head on contrastenhanced 3D-FLAIR imaging is associated with papilledema in patients with idiopathic intracranial hypertension.
\end{abstract}

MATERIALS AND METHODS: A retrospective review was conducted from 2012 to 2015 of patients with clinically diagnosed idiopathic intracranial hypertension and age- and sex-matched controls who had MR imaging with contrast-enhanced 3D-FLAIR. Two neuroradiologists graded each optic nerve/optic nerve head on a scale of $0-3$. This grade was then correlated with the Frisén Scale, an ophthalmologic scale used for grading papilledema from 0 (normal) to 5 (severe edema). To estimate the correlation between the MR imaging and Frisén scores, we calculated the Kendall $\tau$ coefficient.

RESULTS: Forty-six patients ( 3 men, 43 women) with idiopathic intracranial hypertension and 61 controls ( 5 men, 56 women) with normal findings on MR imaging were included in this study. For both eyes, there was moderate correlation between the 2 scales (right eye: $\tau=0.47$; 95\% Cl, 0.31-0.57; left eye: $\tau=0.38 ; 95 \% \mathrm{Cl}, 0.24-0.49$ ). Interreader reliability for MR imaging scores showed high interreader reliability (right eye: $\kappa=0.76 ; 95 \% \mathrm{Cl}, 0.55-0.88$; left eye: $\kappa=0.87 ; 95 \% \mathrm{Cl}, 0.78-0.94$ ). Contrast-enhanced 3D-FLAIR imaging correlates with the Frisén Scale for moderate-to-severe papilledema and less so for mild papilledema.

CONCLUSIONS: Hyperintensity of the optic nerve/optic nerve head on contrast-enhanced 3D-FLAIR is sensitive for the detection of papilledema in patients with idiopathic intracranial hypertension, which may be useful when prompt diagnosis is crucial.

ABBREVIATIONS: $\mathrm{CE}=$ contrast-enhanced; $\mathrm{ICP}=$ intracranial pressure; $I \mathrm{IH}=$ idiopathic intracranial hypertension; $\mathrm{OD}=$ right eye; $\mathrm{ON}=\mathrm{optic}$ nerve; $\mathrm{ONH}=\mathrm{optic}$ nerve head; OS = left eye

diopathic intracranial hypertension (IIH) is an uncommon neurologic condition characterized by elevated intracranial pressure (ICP), which is associated with headaches, diplopia, and papilledema. In rare cases, vision loss has been described in IIH; in cases of fulminant IIH, this deficit has been documented to occur within days of symptom onset. ${ }^{1}$ Vision loss, however, can be halted or even reversed if detected in time, making prompt diag-

Received August 2, 2018; accepted after revision November 23

From the Departments of Radiology (E.G., J.-P.J.Y., T.A.K.), Ophthalmology (R.K., N.M., Y.C.), and Psychiatry (J.-P.J.Y.), University of Wisconsin School of Medicine and Public Health, Madison, Wisconsin; and Department of Biostatistics and Medical Informatics (C.L.), Department of Biomedical Engineering (J.-P.J.Y.), College of Engineering, and Neuroscience Training Program (J.-P.J.Y.), Wisconsin Institutes for Medical Research, University of Wisconsin-Madison, Madison, Wisconsin.

J-P.J.Y. was supported by the University of Wisconsin School of Medicine and Public Health and the Department of Radiology, the Brain and Behavior Research Foundation Young Investigator Grant, and by the Clinical and Translational Science Award program, through the National Institutes of Health National Center for Advancing Translational Sciences, grant ULTR002373.

The content is solely the responsibility of the authors and does not necessarily represent the official views of the National Institutes of Health. nosis of IIH critical. ${ }^{2}$ IIH is diagnosed clinically using the updated Modified Dandy Criteria, the clinical criterion standard that consists of the following: papilledema or other signs and symptoms related to generalized increased ICP, elevated ICP with normal CSF composition, and a neuroimaging study performed to exclude a cause of elevated ICP. ${ }^{1}$ The severity of papilledema in patients with IIH is graded by the Frisén Scale, the ophthalmologic scale for papilledema severity, which ranges from 0 to 5 and denotes normal, very early, early, moderate, marked, and severe papilledema. ${ }^{3-5}$

In contrast to the clinical diagnosis of $\mathrm{IIH}$, the neuroimaging diagnosis of IIH remains a diagnosis of exclusion. Historically, the role of imaging in IIH has been to exclude secondary causes of

Please address correspondence to Tabassum A. Kennedy, MD, Department of Radiology, Division of Neuroradiology, University of Wisconsin School of Medicine and Public Health, 600 Highland Ave, Madison, WI 53792-3252; e-mail: tkennedy@ uwhealth.org; @tabby_kennedy

- - Indicates open access to non-subscribers at www.ajnr.org

http://dx.doi.org/10.3174/ajnr.A5937 
increased ICP in patients with clinically diagnosed papilledema. ${ }^{3}$ However, patients presenting with visual changes or other signs of increased ICP have undergone imaging before funduscopic examination. ${ }^{3}$ This has created an opportunity for neuroimaging to serve as a primary diagnostic tool for clinically suspected IIH. Several radiologic findings of papilledema and IIH have been previously described, including flattening of the globe, protrusion of the optic nerve $(\mathrm{ON})$, a partially empty sella, and transverse sinus stenosis. $^{6-12}$ Postcontrast enhancement of the optic nerve head $(\mathrm{ONH})$ is also a known finding in IIH. ${ }^{6,10}$ Additionally, hyperintensity of the $\mathrm{ONH}$ on diffusion-weighted imaging has also been described. ${ }^{3}$ However, DWI has several intrinsic limitations, which may restrict its use in the diagnosis of IIH. DWI is typically performed with thick sections limiting the visualization of the optic nerve and thereby decreasing the overall sensitivity of the imaging study; additionally, field distortions and other artifacts often seen with DWI in the region of the orbit further limit the sensitivity of DWI for the detection of ONH signal abnormalities. ${ }^{3}$

In the catalogue of descriptions of the radiologic findings of $\mathrm{IIH}$, the MR imaging pulse sequences used in the previous studies are widely varying, with no study to date systematically evaluating the potential role of contrast-enhanced (CE) 3D-FLAIR imaging for the sensitive and specific detection of IIH. Hyperintensity of the ON on CE FLAIR imaging has been used previously in evaluating other abnormalities of the optic nerve, including neuropathy, neuritis, and atrophy. ${ }^{13,14}$ We present the first systematic investigation of CE 3D-FLAIR hyperintensity for the evaluation of the $\mathrm{ON}$ and $\mathrm{ONH}$ for the detection of papilledema in patients with IIH and also present corroborating clinical data that demonstrate both the sensitivity and specificity of our neuroimaging findings.

\section{MATERIALS AND METHODS Subject Population and Controls}

This retrospective study was approved by the University of Wisconsin-Madison institutional review board. A retrospective chart review was performed on consecutive patients with known disease seen in our neuro-ophthalmology clinic between January 2012 and December 2015. The inclusion criteria were patients who had been diagnosed with IIH by a staff neuro-ophthalmologist using the Modified Dandy Criteria and who also had contemporaneous contrast-enhanced MR imaging of the brain with $\mathrm{CE}$ 3D-FLAIR imaging. The severity of papilledema in the subject group was graded by a neuro-ophthalmologist using the Frisén Scale, the ophthalmologic scale for papilledema severity, which ranges from 0 to 5 and denotes normal, very early, early, moderate, marked, and severe papilledema. ${ }^{3-5}$

Age- and sex-matched control subjects were identified sequentially and in chronologic order from a list of MR imaging studies of patients without IIH, retrieved by conducting a text search of the Radiology Information System of our institution. The search criteria for the text search included the desired age \pm 2 years, sex parameters, the key words "unremarkable study" in the radiology report, and completion of the study between January 2012 and December 2015. A subsequent chart review was then performed on all identified control patients to further exclude control subjects with a history of intracranial pathology, increased
Table 1: Demographic and clinical information of study and control cohorts

\begin{tabular}{lcc}
\hline & $\begin{array}{c}\text { Study Cohort } \\
(\boldsymbol{n}=\mathbf{4 6})\end{array}$ & $\begin{array}{c}\text { Control Cohort } \\
(\boldsymbol{n}=\mathbf{6 1})\end{array}$ \\
\hline $\begin{array}{l}\text { Mean age (yr) } \\
\text { Sex }\end{array}$ & $29.6 \pm 11$ & $30.9 \pm 11.7$ \\
$\quad$ Male & 3 & 5 \\
$\quad \begin{array}{l}\text { Female } \\
\text { Frisén Scale papilledema } \\
\quad \text { grade OD (mean) }\end{array}$ & $2.32 \pm 1.3$ & Not available \\
$\begin{array}{c}\text { Frisén Scale papilledema } \\
\quad \text { grade OS (mean) }\end{array}$ & $2.32 \pm 1.3$ & Not available \\
\hline
\end{tabular}

ICP, a history of neurologic surgery, or any other history to suggest a possible diagnosis of IIH. This chart review included any clinic notes from the ordering physician as well as any neurology, neurosurgery, or ophthalmology notes if applicable. Clinical and demographic data were collected for all patients with IIH and controls (Table 1). All control patients were considered negative controls and were presumed to have normal funduscopic examination findings, given that there was no indication in the patient chart that the controls met the Modified Dandy Criteria.

\section{Brain MR Imaging Acquisition}

MR imaging was performed on 1.5T and 3T MR imaging scanners (GE Optima MR450w, GE Signa HDxt, GE Discovery MR750w; GE Healthcare, Milwaukee, Wisconsin). An 8-channel receiver head coil was used for image acquisition. All MR imaging contained the following sequence: contrast-enhanced 3D sagittal T2weighted FLAIR images acquired on $1.5 \mathrm{~T}$ and $3 \mathrm{~T}$ magnets. The sagittal 3D-FLAIR sequence was performed as the initial sequence following the intravenous contrast injection of 0.1 $\mathrm{mmol} / \mathrm{kg}$ of gadobenate dimeglumine (MultiHance; Bracco Diagnostics, Princeton, New Jersey). Coronal and axial reconstructions were subsequently created. The sagittal, coronal, and axial series were available for image review.

\section{Neuroimaging Evaluation}

A grading scheme for evaluation of the $\mathrm{ON}$ and $\mathrm{ONH}$ on $\mathrm{CE}$ 3D-FLAIR imaging was modeled on the 3- and 4-point scales used in previous studies to evaluate hyperintensity. ${ }^{3,6}$ The grading scale is as follows: $0=$ normal; $1=$ mild hyperintensity of the $\mathrm{ON}$ without involvement of the $\mathrm{ONH} ; 2=$ moderate hyperintensity of the $\mathrm{ON}$ and $\mathrm{ONH}$ as well as mild inversion of the $\mathrm{ONH}$; and $3=$ marked hyperintensity of the $\mathrm{ON}$ and $\mathrm{ONH}$ with severe inversion of the ONH (Fig 1). The degree of hyperintensity was ultimately a subjective evaluation of optic nerve signal intensity in relation to the signal intensity of the temporal lobe white matter. Two experienced neuroradiologists with Certificates of Added Qualification, blinded to clinical and demographic data, evaluated the signal intensity of the ON and ONH on CE 3D-FLAIR imaging for both patients with IIH and controls. The images for both the patients with IIH and controls were intermixed and presented to each blinded neuroradiologist in random order. Each ON was evaluated independently and was individually scored with the aforementioned grading scale. An ophthalmologic diagnosis of IIH using the Modified Dandy Criteria was viewed as the criterion standard; therefore, both the true presence and the true severity of the disease were determined by the ophthalmologic examination. 

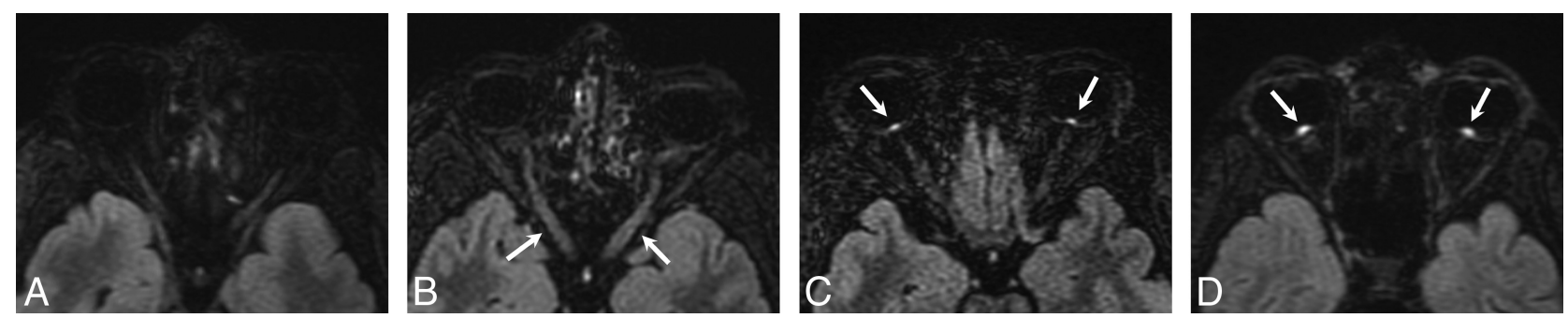

FIG 1. CE 3D-FLAIR images obtained in 4 different patients illustrate the grades of edema seen within the optic nerve and optic nerve head on MR imaging: $0=$ normal $(A), 1=$ mild hyperintensity of the $\mathrm{ON}$ without involvement of the $\mathrm{ONH}$ (arrows, $B$ ), $2=$ moderate hyperintensity of the $\mathrm{ONH}$ as well as mild inversion of the $\mathrm{ONH}$ (arrows, $C$ ), and $3=$ marked hyperintensity of the $\mathrm{ONH}$ with severe inversion of the ONH (arrows, $D$ ).

True disease severity was graded by the neuro-ophthalmologist using the Frisén Scale.

\section{Statistical Methods}

To assess interrater reliability ( 2 readers) for MR imaging on the 0 - to 3-point grading scale and binary scale interpretation, we estimated the Cohen $\kappa$ (quadratically weighted and unweighted, respectively) using the 'psych' package (Version 1.7.8; https:// cran.r-project.org/web/packages/psych/psych.pdf) in R (Version 3.4.3; http://www.r-project.org/) along with $95 \%$ bias-corrected and accelerated-bootstrapped confidence intervals. ${ }^{15}$ For the binary MR imaging scale only, sensitivity and specificity estimates were calculated for each reader along with 95\% Agresti-Coull confidence intervals. To estimate the correlation between MR imaging and Frisén Scale scores, we calculated a nonparametric correlation coefficient (Kendall $\tau$ ) along with the associated $95 \%$ bias-corrected and accelerated-bootstrapped confidence interval (4000 iterations) for each eye. Because optic nerve appearance on MR imaging and fundoscopy is thought to be temporally associated with one another, a sensitivity analysis was conducted via bootstrap simulation to determine whether patients whose MR imaging and funduscopic images were obtained $>30$ days apart were significantly influencing the estimated correlation between MR imaging and Frisén Scale scores.

\section{RESULTS}

\section{Demographic and Clinical Characteristics}

During the study period, 78 patients were clinically diagnosed with IIH on the basis of their clinical funduscopic examination findings by our neuro-ophthalmology colleagues. Following the application of exclusion criteria (no imaging performed, imaging performed at a referring facility, imaging without CE 3D-FLAIR, date of imaging outside the study parameters, and a history of intracranial pathology or instrumentation), 46 patients were included in our study. The study population consisted of 3 men and 43 women with a mean age of $29.6 \pm 11$ years. The incidence of $\mathrm{IIH}$ is significantly higher among women, especially young women and women with obesity. That disparity is reflected therefore in both our patient population and also in our control sample. Frisén Scale scores for the study patients ranged from 0 to 5 , with a histogram pictured in Fig 2. Sixty-one patients with normal findings on MR imaging were included in this study. The control population consisted of 5 men and 56 women, with a mean age of $30.9 \pm 11.7$ years. The indications for MR imaging in these patients were the following: headache $(n=26 ; 42.6 \%)$, inner ear

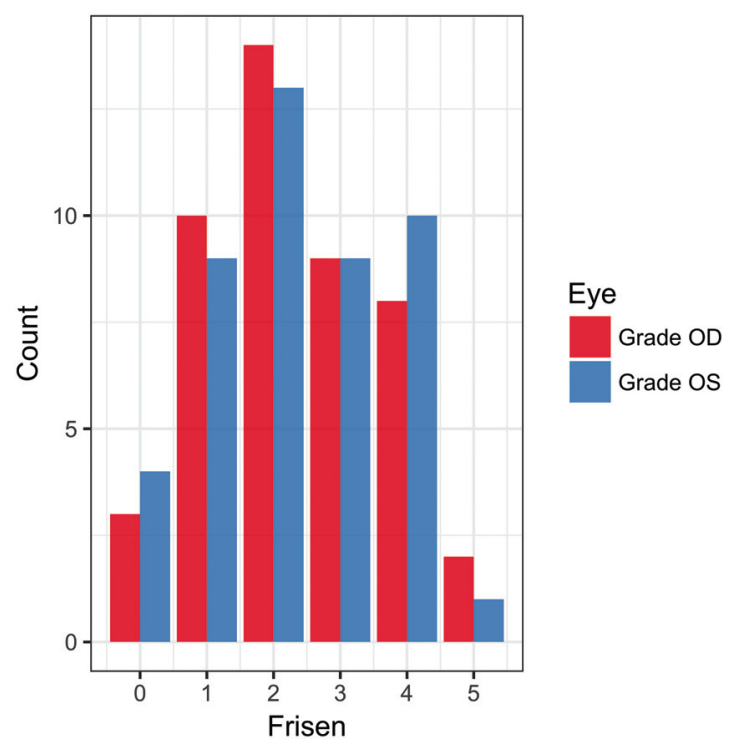

FIG 2. Histogram of observed Frisén Scale scores in the patient group for both OD and OS.

symptoms such as hearing loss or vertigo $(n=11 ; 18.0 \%)$, nonspecific neurologic symptoms such as confusion $(n=5 ; 8.2 \%)$, vision changes $(n=4 ; 6.6 \%)$, neuropathies $(n=4 ; 6.6 \%)$, screening for masses in oncology or patients with transplants $(n=3$; $4.9 \%)$, trauma $(n=2 ; 3.3 \%)$, pituitary symptoms $(n=2 ; 3.3 \%)$, seizures $(n=2 ; 3.3 \%)$, and anosmia $(n=1 ; 1.6 \%)$.

\section{ON and ONH CE FLAIR Imaging Evaluation}

Following blinding of clinical and demographic data, the $\mathrm{ON}$ and $\mathrm{ONH}$ in each patient (IIH and controls) were independently evaluated by both readers. MR imaging findings were considered positive if given any nonzero score on the scale in our study. Each reader found that there was a greater incidence of hyperintensity of the ON/ONH on CE 3D-FLAIR imaging among the patients with IIH than among the control group. For reader 1, hyperintensity of at least 1 ON was $84.4 \%$ sensitive (95\% CI, $75.7 \%-90.4 \%$ ) and $100 \%$ specific ( $95 \%$ CI, $96.3 \%-100 \%$ ) for the presence of papilledema. The correlation between reader l's evaluation of the papilledema grade as measured on the scale of our study and the papilledema grade per the neuro-ophthalmologic Frisén Scale was as follows: right eye (OD): $\tau=0.48 ; 95 \% \mathrm{CI}, 0.32-0.61$; left eye (OS): $\tau=0.43$; $95 \% \mathrm{CI}, 0.24-0.57$. For reader 2 , hyperintensity of at least $1 \mathrm{ON}$ was $77.1 \%$ sensitive $(95 \% \mathrm{CI}, 67.7 \%-84.4 \%)$ and $87.7 \%$ specific (95\% CI, $80.6 \%-92.5 \%$ ) for the presence of papilledema. The correlation between reader 2's evaluation of the 

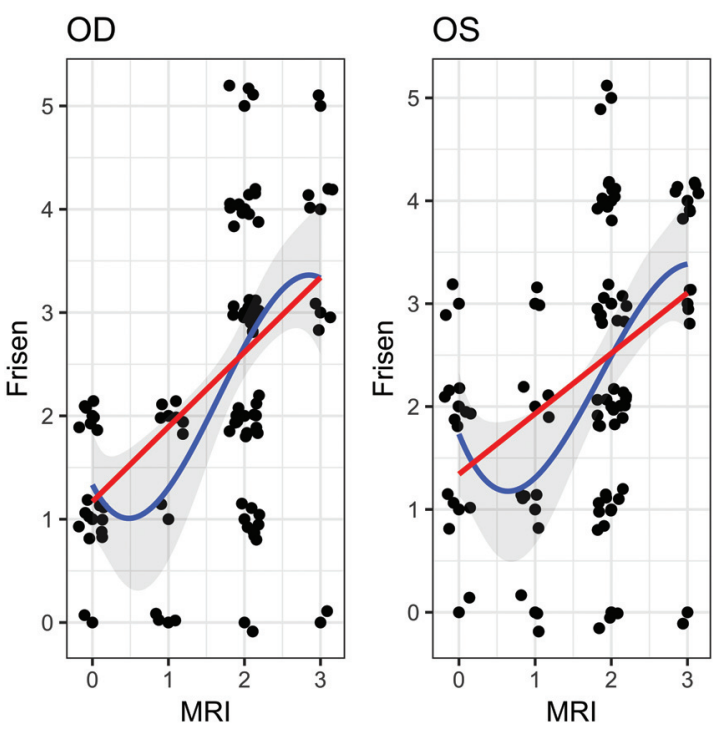

FIG 3. Measured Frisén Scale and MR imaging scores for patients positive for $\mathrm{IIH}$ (with added jitter) for both OD and OS. The blue line represents the estimated cubic polynomial of best fit (with a 95\% confidence band) and the red line represents the estimated linear regression.

\begin{tabular}{|c|c|}
\hline & $P$ Value \\
\hline $\begin{array}{l}T_{\text {reader } 1 \mathrm{OD}}-T_{\text {reader } 1 \mathrm{OD}}^{\prime} \\
T_{\text {reader } 1 \mathrm{OS}}-T_{\text {reader } 1 \mathrm{OS}}^{\prime} \\
T_{\text {reader } 2 \mathrm{OD}}-T_{\text {reader } 2 \mathrm{OD}}^{\prime} \\
T_{\text {reader } 2 \mathrm{OS}}-T_{\text {reader } 2 \mathrm{OS}}^{\text {rea }}\end{array}$ & $\begin{array}{l}.453 \\
.553 \\
.612 \\
.533 \\
\end{array}$ \\
\hline
\end{tabular}

Note: $-T$ indicates the study population correlation coefficient; $T$ ', the restricted group correlation coefficient.

papilledema grade as measured on the scale of our study and the papilledema grade per the neuro-ophthalmologic Frisén Scale was as follows: OD: $\tau=0.46$; 95\% CI, 0.13-0.61; OS: $\tau=0.33$; $95 \%$ CI, 0.05-0.50. When we combined the scores from both readers, the correlation between the 2 scales was as follows: OD: $\tau=0.47$; 95\% CI, 0.31-0.57; OS: $\tau=0.38$; 95\% CI, $0.24-0.49$ (Fig 3).

Interreader reliability for the detection of abnormal signal intensity within the ON of those with IIH was assessed using a Cohen $\kappa$ unweighted score and showed moderate-to-substantial agreement between the 2 readers (OD: $\kappa=0.73$; 95\% CI, $0.36-$ 0.92; OS: $\kappa=0.76 ; 95 \% \mathrm{CI}, 0.36-0.94)$. Interreader reliability for MR imaging grades on the scale of our study was assessed using the Cohen unweighted $\kappa$ (quadratic) and showed substantial-toalmost-perfect agreement between the 2 readers (OD: $\kappa=0.76$; 95\% CI, 0.55-0.88; OS: $\kappa=0.87$; 95\% CI, 0.78-0.94).

The range of time between the funduscopic evaluation and MR imaging in our study population was $0-86$ days. Subgroup analysis conducted on a restricted group of patients with IIH who had undergone imaging or fundoscopy within 30 days of one another did not yield any statistically significant differences in results. The $P$ values for the differences in the correlation coefficients between the study population and the restricted group are listed in Table 2.

\section{DISCUSSION}

In this retrospective study, we sought to determine the sensitivity and specificity of CE 3D-FLAIR imaging to detect papilledema in patients with IIH and to further quantify the relationship between our proposed imaging measures of papilledema and the Frisén Scale, a clinical neuro-ophthalmologic grading scale of papilledema severity. Our results demonstrate that postcontrast FLAIR hyperintensity of the $\mathrm{ON}$ and $\mathrm{ONH}$ is strongly correlated with a clinical diagnosis of IIH and that postcontrast FLAIR hyperintensity of at least $1 \mathrm{ON}$ is at once a robust, sensitive, and specific sign of papilledema. In an extension of our work, we present additional evidence of a positive correlation between the Frisén Scale and our proposed imaging measures of papilledema, highlighting both the clinical utility of our study and its translational potential.

3D-FLAIR imaging affords several built-in advantages when imaging the $\mathrm{ON}$ because it addresses many of the well-known challenges that come with optic nerve imaging. Optimal imaging of the optic nerve includes suppression of the orbital fat surrounding the optic nerve to circumvent high signal intensity and chemical shift artifacts, and fluid suppression to suppress the high signal from the CSF surrounding the optic nerve. Both fluid and fat suppression can be easily achieved with FLAIR imaging. ${ }^{16}$ While FLAIR can be acquired with a $2 \mathrm{D}$ or 3D acquisition, 3DFLAIR has been shown to be superior to 2D-FLAIR for imaging optic neuropathy, highlighted by its ability to generate multiplanar reconstructions to follow tortuous ONs as well as the high spatial resolution afforded by $3 \mathrm{D}$-FLAIR imaging. ${ }^{16}$ Because FLAIR imaging is also a standard feature of many brain imaging protocols, findings related to IIH in the optic nerve may be detected even if clinical concern has not yet prompted a request for orbit-specific imaging. Other sequences such as steady-state free precession sequences (eg, FIESTA or CISS) have been shown to adequately detect optic nerve findings seen with $\mathrm{IIH}$, but unlike FLAIR, are often supplementary sequences performed for a specific indication.

Our neuroimaging findings of high postcontrast FLAIR signal in the optic nerve and optic nerve head are also consistent with prevailing biologic and biomechanical models of elevated intracranial pressure in which elevated intracranial pressure leads to axoplasmic stasis and subsequent neurotoxicity. ${ }^{17-22}$ Axoplasmic stasis is a phenomenon of interrupted axonal transport that results in swelling of nerve fibers, organelle aggregation, and subsequent metabolic waste accumulation leading to cytotoxicity. ${ }^{22}$ Elevated intracranial pressure is hypothesized to lead to the mechanical transduction of increased pressure along the optic nerve sheath that leads to compression of the surrounding vasculature and the resultant venous stasis. ${ }^{22}$ This venous stasis potentially accounts for the enhancement often seen at the optic nerve head in IIH. ${ }^{10,23}$ Other similar hypotheses and models propose the development of axonal ischemia occurring in the setting of increased ICP, which mechanically compresses the ciliary circulation. ${ }^{3,24}$ It is likely that multiple factors contribute to the edema and enhancement seen at the level of the optic nerve head.

Several studies in the literature have shown increased conspicuity of changes related to optic neuropathy in patients with suspected optic neuritis using a CE FLAIR technique. ${ }^{13,14}$ They theorized that the increased conspicuity on CE FLAIR is predom- 
inantly related to T2/FLAIR signal prolongation, with alternative potential contributions from enhancing scar, ongoing demyelination, or other mechanisms resulting in blood-brain barrier breakdown. ${ }^{13}$ We hypothesized that the observed hyperintensity within the $\mathrm{ON}$ and $\mathrm{ONH}$ in patients with IIH is related to synergistic effects between the edematous changes within the $\mathrm{ON}$ and $\mathrm{ONH}$ manifested on FLAIR and concomitant venous stasis, which may also be seen as enhancement, thus likely contributing to the observed increased sensitivity of this imaging sign on CE 3D-FLAIR. Venous status may result in breakdown of the blood-retinal barrier, ultimately leading to enhancement that is isolated to the optic nerve head in patients with IIH. ${ }^{10,23}$ It is unknown, however, how the timing of contrast relative to the acquisition of the $3 \mathrm{D}$ FLAIR sequence affects the signal hyperintensity appreciated in the optic nerve and optic nerve head in these patients. At our institution, the 3D-FLAIR sequence is performed as the initial sequence after the administration of gadobenate dimeglumine (MultiHance). To fully understand the relative contribution of the T2 effects seen on FLAIR versus postcontrast effects, future investigation is needed to evaluate the sensitivity and specificity of 3D-FLAIR without contrast in patients with IIH. ${ }^{10,25}$

While CE 3D-FLAIR imaging shows evidence of papilledema in patients with IIH, with a reasonable amount of sensitivity and specificity, the correlation between the Frisén Scale and our scale was only moderately positive. The correlation differs with the different degrees of severity, with a more linear correlation for cases of moderate-to-severe papilledema and no correlation for cases of mild papilledema. This discrepancy could be partially due to the inherent subjectivity of our scale and reader disagreement on the subtleties of mild hyperintensity in the optic nerve versus normal signal. Image noise present in the axial and coronal reconstructions may also be a contributing factor to the discrepancy observed between the readers in cases of mild papilledema, but it is likely not a confounding factor in more severe cases of papilledema. The variability and discrepancy in mild states of disease have also been shown in previous studies. ${ }^{3}$ Viets et al, ${ }^{3}$ who investigated increased diffusion signal on DWI as an indicator of papilledema in patients with $\mathrm{IIH}$, also found that their sign became more sensitive as the Frisén Scale grade increased. This finding suggests that hyperintensity of the optic nerve on FLAIR imaging cannot be used for a definitive diagnosis of all severities of IIH but may be useful for the detection of more severe IIH.

Whether the papilledema grade correlates with visual outcome is controversial. ${ }^{4,26}$ The papilledema grade has not been found to be correlated with the severity of visual impairment. ${ }^{27,28}$ However, some studies have also found that only patients who present with "obvious" papilledema on physical examination ever develop clinically meaningful visual impairment. ${ }^{28}$ In this subset of patients, the evidence suggests that the papilledema grade does correlate with the amount of vision loss at higher grades of papilledema. ${ }^{28,29}$ Additionally, patients who present with vision loss at the time of diagnosis also tend to have poor visual outcomes. ${ }^{24}$ This finding would suggest that the most useful diagnostic imaging study would be one that detects papilledema in patients who would ultimately be found to have moderate-to-severe papilledema on funduscopic examination.

The limitations of our study are those primarily related to the retrospective study design. It was not possible to obtain funduscopic photographs for the control group; therefore, the controls were assumed to have negative funduscopic examination findings (Frisén Scale $=0$ ). Therefore, despite the best attempts to exclude control patients with a history of $\mathrm{IIH}$, intracranial pathology, or instrumentation, a chart review cannot fully ensure that all control patients were true-negative controls. Another potential limitation is that a designated temporal relationship between a funduscopic photograph and MR imaging was not one of the inclusion criteria for this study. However, a subgroup analysis of patients who underwent MR imaging and had funduscopic photos within 30 days of one another did not show markedly different results than those presented here. Another limitation of this study is that the FLAIR images were not compared with postcontrast T1-weighted sequences. This limitation was due to the heterogeneity in neuroimaging protocols used in both the study and control populations, leading to a variety of different postcontrast sequences performed, thus limiting the ability to directly compare sequences.

\section{CONCLUSIONS}

Herein, we present the first application of CE 3D-FLAIR imaging for the detection of papilledema in patients with clinically diagnosed IIH and demonstrate that hyperintensity of the optic nerve on CE 3D-FLAIR is a sensitive and specific sign for papilledema in patients with IIH. Additionally, we show a moderately positive correlation between the degree of hyperintensity and the severity of papilledema as measured on the neuro-ophthalmologic Frisén Scale and demonstrate that this correlation is strongest in patients with severe papilledema. Therefore, significant hyperintensity of the optic nerve on CE 3D-FLAIR imaging is a useful detection tool for IIH in high-risk patients in whom prompt diagnosis is critical but neuro-ophthalmologic evaluation is not readily available.

Disclosures: John-Paul J. Yu-RELATED: Grant: National Institutes of Health KL2 Award, Brain and Behavior Research Foundation. * Money paid to the institution.

\section{REFERENCES}

1. Shaikh AG, Bates JH, Yeates SW, et al. Fulminant idiopathic intracranial hypertension. JAMA Neurol 2013;70:937-38 CrossRef Medline

2. Bidot S, Bruce BB. Update on the diagnosis and treatment of idiopathic intracranial hypertension. Semin Neurol 2015;35: 527-38 CrossRef Medline

3. Viets R, Parsons M, Van Stavern G, et al. Hyperintense optic nerve heads on diffusion-weighted imaging: a potential imaging sign of papilledema. AJNR Am J Neuroradiol 2013;34:1438-42 CrossRef Medline

4. Wall M, Kupersmith MJ, Kieburtz KD, et al; NORDIC Idiopathic Intracranial Hypertension Study Group. The idiopathic intracranial hypertension treatment trial: clinical profile at baseline. JAMA Neurol 2014;71:693-701 CrossRef Medline

5. Frisén L. Swelling of the optic nerve head: a backstage view of a staging scheme. J Neuroophthalmol 2017;37:3-6 CrossRef Medline

6. Padhye LV, Van Stavern GP, Sharma A, et al. Association between visual parameters and neuroimaging features of idiopathic intracranial hypertension. J Neurol Sci 2013;332:80 - 85 CrossRef Medline

7. Salvay DM, Padhye LV, Huecker JB, et al. Correlation between papilledema grade and diffusion-weighted magnetic resonance imaging in idiopathic intracranial hypertension. $J$ Neuroophthalmol 2014;34:331-35 CrossRef Medline 
8. Hirfanoglu T, Aydin K, Serdaroglu A, et al. Novel magnetic resonance imaging findings in children with intracranial hypertension. Pediatr Neurol 2015;53:151-56 CrossRef Medline

9. Morris PP, Lachman N, Black DF, et al. Increased curvature of the tentorium cerebelli in idiopathic intracranial hypertension. AJNR Am J Neuroradiol 2017;38:1789-93 CrossRef Medline

10. Degnan AJ, Levy LM. Pseudotumor cerebri: brief review of clinical syndrome and imaging findings. AJNR Am J Neuroradiol 2011;32: 1986-93 CrossRef Medline

11. Agid R, Farb RI, Willinsky RA, et al. Idiopathic intracranial hypertension: the validity of cross-sectional neuroimaging signs. Neuroradiology 2006;48:521-27 CrossRef Medline

12. Morris PP, Black DF, Port J, et al. Transverse sinus stenosis is the most sensitive MR imaging correlate of idiopathic intracranial hypertension. AJNR Am J Neuroradiol 2017;38:471-77 CrossRef Medline

13. Boegel KH, Tyan AE, Iyer VR, et al. Utility of coronal contrast-enhanced fat-suppressed FLAIR in the evaluation of optic neuropathy and atrophy. Eur J Radiol Open 2017;4:13-18 CrossRef Medline

14. Pino-Lopez L, Wenz H, Bohme J, et al. Contrast-enhanced fat-suppressed FLAIR for the characterization of leptomeningeal inflammation in optic neuritis. Mult Scler 2018 Apr 1. [Epub ahead of print] CrossRef Medline

15. Revelle WR (photographer). psych: Procedures for Personality and Psychological Research. 2017. https://www.scholars.northwestern. edu/en/publications/psych-procedures-for-personality-andpsychological-research. Accessed July 262018

16. Aiken AH, Mukherjee P, Green AJ, et al. MR imaging of optic neuropathy with extended echo-train acquisition fluid-attenuated inversion recovery. AJNR Am J Neuroradiol 2011;32:301-05 CrossRef Medline

17. Killer HE, Jaggi GP, Flammer J, et al. Cerebrospinal fluid dynamics between the intracranial and the subarachnoid space of the optic nerve: is it always bidirectional? Brain 2007;130:514-20 CrossRef Medline

18. Jonas JB, Wang NL, Yang DY, et al. Facts and myths of cerebrospinal fluid pressure for the physiology of the eye. Prog Retin Eye Res 2015; 46:67-83 CrossRef Medline

19. Killer HE, Jaggi GP, Miller NR. Papilledema revisited: is its pathophysiology really understood? Clin Exp Ophthalmol 2009;37:444-47 CrossRef Medline

20. Tso MO, Hayreh SS. Optic disc edema in raised intracranial pressure, IV: axoplasmic transport in experimental papilledema. Arch Ophthalmol 1977;95:1458-62 CrossRef Medline

21. Radius RL, Anderson DR. Morphology of axonal transport abnormalities in primate eyes. Br J Ophthalmol 1981;65:767-77 CrossRef Medline

22. Hayreh SS. Pathogenesis of optic disc edema in raised intracranial pressure. Prog Retin Eye Res 2016;50:108-44 CrossRef Medline

23. Manfré L, Lagalla R, Mangiameli A, et al. Idiopathic intracranial hypertension: orbital MRI. Neuroradiology 1995;37:459-61 CrossRef Medline

24. Corbett JJ, Savino PJ, Thompson HS, et al. Visual loss in pseudotumor cerebri: follow-up of 57 patients from five to 41 years and a profile of 14 patients with permanent severe visual loss. Arch Neurol 1982;39:461-74 CrossRef Medline

25. Brodsky MC, Vaphiades M. Magnetic resonance imaging in pseudotumor cerebri. Ophthalmology 1998;105:1686-93 CrossRef Medline

26. Obi EE, Lakhani BK, Burns J, et al. Optic nerve sheath fenestration for idiopathic intracranial hypertension: a seven-year review of visual outcomes in a tertiary centre. Clin Neurol Neurosurg 2015;137: 94-101 CrossRef Medline

27. Trobe JD. Papilledema: the vexing issues. J Neuroophthalmol 2011; 31:175-86 CrossRef Medline

28. Wall M. Idiopathic intracranial hypertension. Neurol Clin 2010;28: 593-617 CrossRef Medline

29. Wall M, White WN 2nd. Asymmetric papilledema in idiopathic intracranial hypertension: prospective interocular comparison of sensory visual function. Invest Ophth Vis Sci 1998;39:134-42 Medline 